\title{
Alterstice
}

Revue internationale de la recherche interculturelle

International Journal of Intercultural Research

Revista International de la Investigacion Intercultural

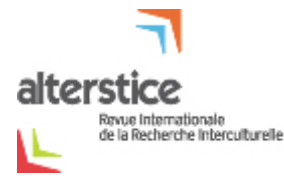

\section{Migrations et " nouvelles mobilités " : regards d'élèves et d'enseignants dans une école de langue française en Ontario (Canada)}

\section{Diane Farmer}

Volume 6, numéro 1, 2016

Prendre en compte la diversité à l'école

URI : https://id.erudit.org/iderudit/1038283ar

DOI : https://doi.org/10.7202/1038283ar

Aller au sommaire du numéro

Éditeur(s)

Alterstice

ISSN

1923-919X (numérique)

Découvrir la revue

Citer cet article

Farmer, D. (2016). Migrations et « nouvelles mobilités » : regards d'élèves et d'enseignants dans une école de langue française en Ontario (Canada).

Alterstice, 6(1), 105-119. https://doi.org/10.7202/1038283ar

\section{Résumé de l'article}

L’attention portée à la mobilité géographique - déplacements quotidiens, tourisme, migration, immigration, mouvements transnationaux, les configurations sont nombreuses - permet d'ouvrir de nouveaux chantiers en ce qui a trait à la construction de sens dans les relations à l'altérité. L'école est sans équivoque l'un des principaux lieux où se manifestent les pratiques plus mobiles des familles. Comment les élèves et les enseignants d'une école caractérisée par des mouvements rapides de population donnent-ils sens à leur parcours migratoire, en lien avec celui des autres acteurs de l'école, et dans l'esprit de vivre dans un monde en commun? Comment l'école s'adapte-t-elle au renouvellement continu des familles? Cet article présente la réflexion d'élèves, d'enseignants et de la direction d'une école de langue française de quartier populaire en Ontario. Il met en lien les représentations d'acteurs à partir de récits et entrevues dans le cadre des multiples mouvements migratoires caractéristiques du monde d'aujourd'hui. L'analyse vise à remettre en question le travail de classification entre locaux et migrants, en cherchant à mettre en valeur le travail de co-construction d'un univers de sens réalisé par les enfants et les adultes d'une école primaire. 


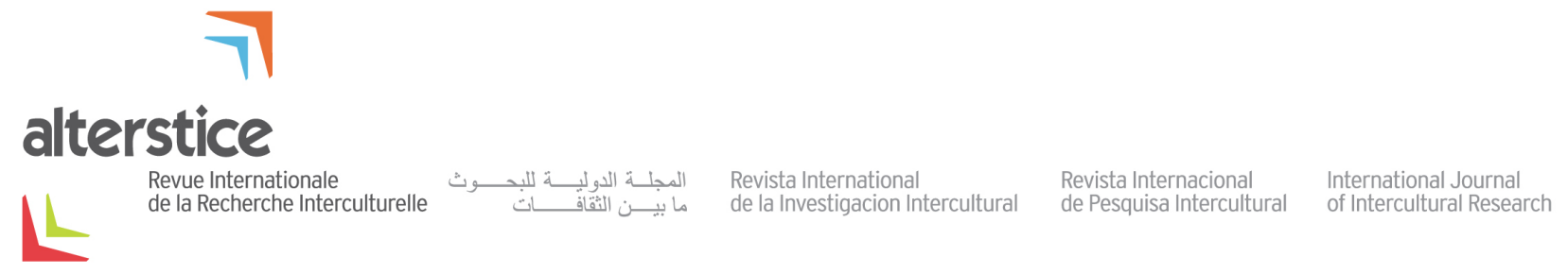

ARTICLE THÉMATIQUE

\title{
Migrations et " nouvelles mobilités » : regards d'élèves et d'enseignants dans une école de langue française en Ontario (Canada)
}

\author{
Diane Farmer ${ }^{1}$
}

\section{Résumé}

L'attention portée à la mobilité géographique - déplacements quotidiens, tourisme, migration, immigration, mouvements transnationaux, les configurations sont nombreuses - permet d'ouvrir de nouveaux chantiers en ce qui a trait à la construction de sens dans les relations à l'altérité. L'école est sans équivoque l'un des principaux lieux où se manifestent les pratiques plus mobiles des familles. Comment les élèves et les enseignants d'une école caractérisée par des mouvements rapides de population donnent-ils sens à leur parcours migratoire, en lien avec celui des autres acteurs de l'école, et dans l'esprit de vivre dans un monde en commun? Comment l'école s'adaptet-elle au renouvellement continu des familles? Cet article présente la réflexion d'élèves, d'enseignants et de la direction d'une école de langue française de quartier populaire en Ontario. II met en lien les représentations d'acteurs à partir de récits et entrevues dans le cadre des multiples mouvements migratoires caractéristiques du monde d'aujourd'hui. L'analyse vise à remettre en question le travail de classification entre locaux et migrants, en cherchant à mettre en valeur le travail de co-construction d'un univers de sens réalisé par les enfants et les adultes d'une école primaire.

\author{
Rattachement de l'auteure \\ ${ }^{1}$ University of Toronto, Toronto, Canada
}

\section{Correspondance}

diane.farmer@utoronto.ca

\section{Mots clés}

enfance; jeunesse; mobilité; migration: élèves; enseignants

\section{Pour citer cet article}

Farmer, D. (2016). Migrations et "nouvelles mobilités ": regards d'élèves et d'enseignants dans une école de langue française en Ontario (Canada). Alterstice, 6(1), 105-120. 


\section{Introduction}

Le Canada est un pays à deux langues officielles, le français et l'anglais. Ce statut juridique particulier a servi d'appui au développement d'un réseau d'enseignement dans la langue de la minorité (l'anglais au Québec et le français au sein des autres provinces canadiennes). C'est ainsi qu'en Ontario, on compte près de 450 écoles de langue française de la maternelle à la $12^{\mathrm{e}}$ année (la $12^{\mathrm{e}}$ année marquant la fin du secondaire) qui coexistent avec le réseau des écoles de la majorité (de langue anglaise). Il est aussi possible de poursuivre des études postsecondaires dans trois établissements collégiaux et six universités ontariennes. La population francophone est historiquement distribuée entre les régions $\mathrm{du}$ nord, de l'est et du sud de l'Ontario. On y dénombre actuellement 611550 francophones, ce qui représente $4,8 \%$ de la population ontarienne (Statistique Canada, 2012). La population franco-ontarienne est diverse, ne se limitant pas aux familles d'origine canadienne-française et acadienne, et elle accueille depuis de nombreuses années des familles d'Afrique, d'Asie, du Moyen-Orient et d'Europe. À ce type de migration s'ajoutent aujourd'hui des mouvements de population plus variés et individualisés, que ce soit au travers des modèles de migration des familles ou encore des déplacements entre régions au sein des provinces canadiennes. Le ministère de l'Éducation de l'Ontario a publié en 2009 la Stratégie ontarienne d'équité et d'éducation inclusive (MEO, 2009), une déclaration de politique qui insiste sur l'obligation faite aux enseignants d'adopter des pratiques pédagogiques affirmant la pluralité des identités de leurs élèves.

La réflexion proposée ici présente les résultats d'une étude ethnographique réalisée en Ontario en contexte scolaire francophone minoritaire. L'enquête visait à explorer comment de jeunes élèves vivant l'expérience de la mobilité géographique sur le plan régional ou de la migration internationale, en fonction de déplacements familiaux complexes, se construisent dans ces mouvements. Comment parlent-ils (s'ils en parlent) de leurs liens avec la francophonie et l'identité francophone ? Et comment des élèves plus sédentaires, du fait d'être exposés à la mobilité de leur groupe de pairs et des enseignants, sont-ils aussi appelés à se construire dans l'optique du mouvement ? Comment ces jeunes expliquent-ils leur parcours de vie en lien avec l'image d'un univers mondialisé, au sens ici de monde en réseaux?

Plus spécifiquement, il s'agira d'explorer les questions suivantes : comment les élèves d'une école caractérisée par des mouvements rapides de population construisent-ils leurs repères quotidiens et quel sens donnent-ils à l'école comme lieu d'ancrage dans le mouvement ? Comment le personnel de cette école s'adapte-t-il au renouvellement continu des familles? Ce texte va mettre en lien les représentations d'acteurs à partir d'une sélection de récits venant saisir les modalités des relations avec autrui dans le cadre de multiples mouvements migratoires. L'analyse vise à remettre en question la dichotomie entre locaux et migrants au sein de l'école, en cherchant à mettre en valeur le travail de co-construction d'un univers de sens réalisé par les adultes et les enfants d'une école primaire.

\section{Cadre conceptuel}

Les mouvements de population prennent aujourd'hui des formes plus fluides, ce qui nous amène à interroger le découpage classique qui oppose "locaux » et " migrants " dans les études traitant de l'immigration. Élias (1997) posait une question similaire en cherchant à expliquer l'opposition observée entre deux quartiers ouvriers similaires d'une petite ville d'Angleterre. Les chercheurs ont conclu que les différences de perception étaient liées au moment d'arrivée des familles dans le quartier. Cette distinction a ensuite été reprise et intériorisée dans un ordre hiérarchique entre une communauté ouvrière établie d'un côté et un quartier marginal de l'autre (voir aussi Heinich, 2002). Dans le cas de l'immigration, on retrouve aussi un travail de construction par les acteurs sociaux, entre un milieu " qui accueille " de nouvelles personnes et une population "à accueillir ». Cette opposition entre "locaux" et "migrants" relève d'un découpage qui entraîne certains effets d'exclusion. Elle s'estompe difficilement malgré l'effacement de la nouveauté : on reste " migrant » ou fille ou fils de migrants bien après les premières années d'arrivée. Peut-on imaginer toutefois le dépassement d'une telle dichotomie au sein de " sociétés contemporaines mobiles " (Urry, 2005), de sociétés où les acteurs ont à se construire en bonne partie à travers le mouvement ? La conscience qu'ont les acteurs sociaux de vivre dans un monde commun peut-elle aider à brouiller les allégeances identitaires? Nous puiserons dans les travaux de sociologie urbaine, de sociologie de l'éducation et de sociologie de l'enfance pour aborder ce concept de "nouvelles mobilités ». 
Urry (2000) invite les chercheurs à repenser ce qu'on entend par le terme de société, en faisant du mouvement plutôt que de la sédentarité un élément central de l'organisation des pratiques sociales. Il entend par mouvement non seulement les déplacements de population au quotidien, au sein d'une ville, d'une ville (lieu de résidence) à une autre (lieu de travail), ou encore les voyages d'affaires ou de tourisme, les déplacements sur de plus longues périodes, migrations voulues ou contraintes, mais également la circulation d'images et d'information à l'échelle locale, nationale et mondiale. Nous sommes ainsi en présence de mouvements plus individualisés, rapides, circulaires et soutenus par une panoplie de réseaux numériques. Urry met ainsi à l'épreuve la notion de société conçue comme territoire.

II propose un nouveau paradigme en sciences sociales qui met l'accent sur l'analyse de la fluidité des mouvements (Urry, 2000 et 2005 ; Sheller et Urry, 2006). Loin de se limiter à relater les grandes tendances dans les mouvements de population, les chercheurs s'intéressent notamment aux parcours singuliers des acteurs sociaux. On cherche à mieux comprendre comment les pratiques sociales, par exemple les dynamiques familiales, sont reconstituées de manière complexe au travers de nouvelles formes de mobilité. En recueillant les histoires de mobilité, ces dynamiques mettent en valeur l'idée d'une continuité bien plus que celle d'une rupture dans la représentation de parcours migratoires (voir par exemple les travaux de Vatz Laaroussi [2009, 2015] sur les réseaux familiaux et sur les rapports intergénérationnels).

Les nouveaux espaces liés à la mobilité évoquent par ailleurs l'idée d'un rapprochement entre populations jadis isolées les unes des autres. Elles génèrent de nouvelles situations où se pose la réciprocité des liens dans une analyse centrée sur la relation avec autrui (Bourdin, 2005 ; White, 2014). Certains auteurs font remarquer que les jeunes, mêmes sédentaires, sont exposés à la mobilité, notamment dans les rapports qu'ils entretiennent avec d'autres jeunes comme dans la participation aux réseaux de technologie de l'information et de communication qui les rassemblent dans une " culture jeune » mondiale (Faist et Kivisto, 2007; Nsamenang, 2002; Suarez-Orozco et Qin-Hilliard, 2004 ; Suarez-Orozco et Suarez-Orozco, 2008). Il convient alors à nouveau de se demander s'il n'y a pas là une occasion de brouiller la dichotomie entre locaux (le Nous) et migrants (I'Autre qui n'est pas Nous).

À la question de nouveaux espaces s'ajoute une réflexion sur le rôle joué par les infrastructures dans l'étude du mouvement. L'image d'un aéroport est souvent reprise pour évoquer la distance relative entre les villes (on serait plus près des autres régions du monde lorsqu'on vit dans un endroit qui a son propre aéroport). Il en va de même pour comprendre la complexité des interactions au sein de ces espaces particuliers (on ne fait pas qu'attendre son $\mathrm{vol}$, on y travaille, consomme, rencontre d'autres gens qui se déplacent également) (Urry, 2000 et 2005 ; Sheller et Urry, 2006). Mobilité et lieux d'ancrage sont alors des variables indissociables. On ne peut analyser les pratiques de mobilité sans leur contexte et sans tenir compte des réseaux et infrastructures qui rendent possibles de tels mouvements (Appadurai, 1996 ; Fass, 2007 ; Portes, 1999). II s'agit donc d'étudier le mouvement en lien avec les systèmes qui le rendent possible.

Comment aborde-t-on la mobilité en sociologie de l'éducation ? Tout d'abord, il s'agit d'un thème encore peu abordé (Landri et Neumann 2014). Les enquêtes mettent de l'avant le travail de préparation des jeunes, visant à rendre ces derniers compétitifs sur les marchés mondialisés, ou encore dénoncent l'effet réducteur d'une telle perspective (Mitchell, 2003; Weenink, 2008). Ce travail préparatoire qui exerce des pressions sur l'école inclut la nécessité d' " être formé " à l'esprit cosmopolite (être disposé à bouger et capable d'interagir dans divers contextes culturels), de vivre l'expérience d'échanges internationaux et enfin de développer un capital linguistique (Shin, 2012; Song, 2010). Les systèmes d'éducation se seraient donc engagés dans la voie de la formation d'une élite mondialisée. Thamin (2007) évoque à cet égard l'idée d'une nouvelle doxa qui oriente les façons de faire en éducation. Le contexte des nouvelles mobilités nous incite alors à revoir la question de l'inégalité des chances en éducation : I'articulation de nouvelles formes de mobilité peut-elle à la fois offrir de nouvelles possibilités aux acteurs sociaux et devenir source d'inégalité, " avoir partie liée avec une mobilité sociale vers le haut ou vers le bas... » (Urry, 2005, p. 27, voir aussi Kaufmann, 2005)?

Notre enquête s'inscrit par ailleurs dans l'avancement des travaux sur le métier d'élève (Perrenoud, 1995 ; Sirota, 1993) et de la sociologie de l'enfance plus largement (par exemple Bluebond-Langner et Korbin 2007 ; Christensen et James 2008 ; Corsaro, 2005 ; James, 2007 ; Montandon, 1998 ; Prout, 2005 ; Sirota, 1998 et 2006 ). Ce champ de 
recherche a pris forme à partir d'interrogations sur la construction sociologique de l'enfance, la voix de l'enfant et plus récemment la participation d'enfants aux diverses étapes du processus de recherche. On y aborde l'enfance en tant que catégorie sociale et l'enfant en tant qu'acteur social légitime, observateur, comme tous les autres acteurs sociaux, du monde dans lequel il se trouve et sur lequel il agit. L'enfance ne s'étudie pas de façon isolée, mais en relation avec les adultes qui partagent les mêmes espaces, c'est-à-dire dans le cas qui nous intéresse avec les enseignants de l'école. Reconnaissant que les enfants vivent diverses formes d'oppression, les chercheurs s'accordent à dire qu'il existe de fait une pluralité d'enfances.

Alors que les travaux en éducation cités plus haut mettent l'accent sur la préparation des jeunes aux marchés de l'emploi mondialisés, les chercheurs dans le domaine de l'enfance se sont intéressés à l'expérience des enfants et au sens qu'ils donnent à de telles expériences. Ní Laoire, Carpena-Méndez, Tyrrell et White (2010), de même que Ralph et Staeheli (2011), révèlent dans leurs enquêtes comment les jeunes se construisent effectivement dans le mouvement et soulignent l'importance des liens affectifs et identitaires dans cette construction. Un tel recadrage sur l'enfance peut fournir un éclairage nouveau à la question du découpage qui oppose " locaux » et " migrants » en milieu scolaire.

L'analyse présentée ici explore le sens qu'accordent les élèves à leurs parcours de mobilité et aux relations qu'ils développent à partir de mouvements migratoires complexes. On peut alors se demander dans quelle mesure l'expérience d'un parcours mobile (le sien ou celui des élèves de la classe) influence les représentations identitaires, représentation de soi et de l'autre. Les réflexions biographiques commentées abordent le mouvement en mettant de l'avant la complexité des allégeances identitaires. Ils illustrent le travail de recomposition effectué par ces jeunes et leurs enseignants et conduisent ainsi à brouiller les désignations identitaires entre locaux et migrants. Cette étude traite par ailleurs du rôle de l'école en tant qu'institution pouvant faciliter le mouvement des familles. L'école évoque cette idée d'une structure (tel un aéroport) qui attire les mouvements migratoires des familles et qui se voit transformée, par la même occasion, par les mouvements de population (des familles, mais aussi du personnel de l'école). L'enjeu qui nous préoccupe alors est celui de l'inclusion. Comment l'école s'adaptet-elle au renouvellement continu des familles ? Si le mouvement amène de nouvelles possibilités d'action, il peut aussi stigmatiser certaines populations. Nous avançons l'idée qu'un tel enjeu se joue dans le travail de classification entre locaux et migrants. Comment le personnel de l'école négocie-t-il une telle frontière au jour le jour? Qu'en est-il, plus précisément, du découpage entre locaux et migrants ? Quels types de défis l'école doit-elle relever?

\section{Approche méthodologique et contexte de l'étude}

Les chercheurs ont recours en général à l'approche ethnographique lorsqu'il s'agit d'aborder une problématique de recherche à partir d'une perspective visant à rendre compte de la complexité d'une situation sociale, du contexte et du jeu des acteurs. Cette méthode s'avère utile pour revoir les catégories de pensées devenues invisibles. La dichotomie pressentie entre le Nous collectif et l'Autre, l'étranger, le migrant, constitue une typologie construite à partir d'un regard macro-social, un regard alimenté par les documents de politique, par les discours sur les identités nationales ou encore par le biais de grandes enquêtes où populations locales et migrantes sont comparées et reconstruites, notamment en lien aux inégalités sociales dans le domaine de l'emploi, de l'éducation et du logement. Mais qu'en est-il d'une perspective partant du bas, des acteurs eux-mêmes, et de la construction qu'ils font de leur monde social, une construction qui oriente leurs actions ? L'enquête ethnographique, nous précisent Beau et Weber (1997), vise alors à restituer le point de vue d'acteurs absents, en marge des analyses.

Nous avons choisi comme méthodologie de réaliser une enquête multi-sites combinant l'observation prolongée en salle de classe et des entretiens en petits groupes ou individuels. Au-delà de ces techniques classiques dans la réalisation d'ethnographies scolaires, nous avons également mené une enquête intra-site où les jeunes participants ont eu l'occasion de réfléchir au thème de la mobilité en menant leur propre exploration dans leur quartier et à la maison. À cet effet, nous avons intégré des techniques créatives visuelles de recherche, pour offrir ainsi aux jeunes participants des outils méthodologiques leur permettant de partager leur expérience de vie, à partir de moyens sur lesquels ils ont un plus grand contrôle (Farmer et Prasad, 2014). 
Les méthodes créatives visuelles sont généralement utilisées en lien avec une entrevue et d'autres techniques donnant lieu à des témoignages et au partage d'une réflexion, conjointement avec le chercheur (Farmer et Cepin, 2015 ; Rose, 2014). Le recours au dessin réflexif vise par ailleurs à diminuer l'influence du chercheur en offrant un espace d'échange moins directif. Les données recueillies dans cette enquête ont été produites dans cette optique, à partir d'un cadre dialogique réflexif dans lequel les jeunes participants ont discuté de leurs parcours de vie et construit leur récit de vie avec la chercheure et, surtout, entre eux. Il existe de nombreuses techniques visuelles de recherche qui soutiennent les approches réflexives. Nous avons utilisé dans cette enquête à la fois le dessin réflexif et la photographie. Notre analyse traite plus spécifiquement de données recueillies à partir du dessin et des entrevues réalisées avec les jeunes et le personnel enseignant ainsi que la directrice de l'école. Il existe plusieurs techniques de dessin : celle utilisée dans cette enquête a été empruntée de la sociolinguistique (Busch, 2010 ; Castellotti et Moore, 2009; Krumm, 2008) et a consisté à dessiner son portrait de langues. Cet outil a été développé dans le cadre d'études portant notamment sur la langue et le pouvoir, l'éveil aux langues, l'identité et le plurilinguisme. Nous avons repris et adapté l'approche spécifique développée par Busch (2010; Busch, Jardine et Tjoutuku, 2006) qui consiste à prendre le corps comme métaphore pour aborder, dans une perspective biographique, les multiples relations (cognitives, affectives et autres) avec le langage.

Comment donc les enfants construisent-ils leurs repères quotidiens à travers l'expérience de la mobilité géographique et des mouvements migratoires familiaux ? La consigne reçue par les élèves et les enseignants a été la suivante: "Dessine sur la silhouette les langues et les cultures qui t'habitent, des cultures avec lesquelles tu as développé un lien au courant de ta vie ". Tout comme dans le cas de Busch, les participants devaient créer une légende de couleurs afin de préciser leurs catégories de classement. Ils devaient expliquer au fil de la discussion les associations faites avec les parties du corps. La silhouette générique (un petit bonhomme) a été progressivement remplacée dans l'enquête menée sur la mobilité par une représentation numérique des participants ${ }^{1}$.

L'enquête a été réalisée dans cinq salles de classe en Ontario : une classe de $3^{\mathrm{e}}$ année (élèves de 8 ans) dans une école d'immersion française qui a servi d'étude exploratoire donnant lieu à l'expérimentation de techniques visuelles de recherche avec de jeunes élèves et leur enseignante, une classe de $4^{e}$ année (élèves de 9 ans) et une classe de $10^{\mathrm{e}}$ année (élèves de 15 ans) dans une école privée internationale de langue française ainsi que dans une classe de $4^{\mathrm{e}} / 5^{\mathrm{e}}$ année (élèves de 9 et 10 ans) et une classe de $5^{\mathrm{e}} / 6^{\mathrm{e}}$ année (élèves de 10 et 11 ans) dans une école publique de la minorité francophone accueillant des familles à faible revenu. Au total, 125 personnes ont pris part à cette étude, plus des deux tiers étant des élèves. Nous avons interviewé aussi les directions d'école et enseignants ainsi que des parents d'élèves. Les élèves de ces trois établissements sont issus de migrations interprovinciales et/ou internationales. Ils ne disposent pas des mêmes ressources, au sens d'un nouveau type de capital évoqué par Urry (2005) et par Kaufmann (2005). L'analyse qui suivra focalise sur l'école publique francophone accueillant des familles à faible revenu. Il s'agit de l'école primaire l'Hirondelle ${ }^{2}$.

La ville où l'étude a été réalisée se trouve dans la région du sud-ouest de l'Ontario, région où la présence française remonte au début $\mathrm{du} 18^{\mathrm{e}}$ siècle. Elle a un long passé industriel, et la région est devenue au début du $20^{\mathrm{e}}$ siècle la capitale de l'industrie automobile. Aujourd'hui, la ville travaille à rebâtir son économie en se diversifiant, notamment dans les domaines du tourisme et des loisirs, des sciences de la santé et la technologie des énergies renouvelables. Sur le plan linguistique, $72,9 \%$ de la population déclare l'anglais comme langue maternelle, 3,3\% le français et 21,5\% une langue non officielle (Statistique Canada, 2012). Les langues maternelles non officielles les plus courantes sont par ordre décroissant l'arabe (16\%), l'italien $(14,2 \%)$, le chinois $(4,7 \%)$, le polonais $(4,6 \%)$, le serbe $(4,3 \%)$, l'espagnol $(4,2 \%)$, le roumain $(3,3 \%)$, l'allemand $(3,1 \%)$, les langues sémitiques $(3,0 \%)$ et le pendjabi $(3,0 \%)$.

L'école primaire l'Hirondelle est une école publique de langue française ayant ouvert ses portes en 1998. Au moment de l'étude (en 2011), la directrice, également fondatrice de l'école, et cinq membres du personnel

\footnotetext{
${ }^{1}$ C'est le cas pour les élèves et pour les enseignantes de la classe de $4^{\mathrm{e}} / 5^{\mathrm{e}}$ et de $5^{\mathrm{e}} / 6^{\mathrm{e}}$. Les portraits réalisés par les autres enseignants ayant participé à l'étude ainsi que les enseignants d'une autre école ont été faits à partir d'une silhouette générique. Il s'agit donc d'un outil qui a évolué au cours de l'enquête.

${ }^{2}$ Nom fictif. Les noms des participants à l'étude et le nom de l'école sont des pseudonymes.
} 
enseignant (sur 15 enseignants) y travaillent toujours ${ }^{3}$. L'école accueille 215 élèves de familles à revenu modeste, provenant en majorité du Moyen-Orient et dans une moindre mesure d'Afrique, d'Amérique du Sud, d'Europe, de l'Ontario et d'autres régions du Canada. La majorité des élèves parlent arabe à la maison.

\section{Présentation et discussion des résultats}

Nous présentons et discutons des résultats de recherche à partir d'abord de récits d'élèves, dans lesquels se trouvent imbriqués leurs récits familiaux et les propos qu'ils tiennent au sujet de l'école ainsi que sur l'identité francophone. Nous examinons ensuite comment l'école perçoit le contexte de mobilité des familles et comment elle répond aux enjeux de la mobilité dans une école qui accueille des familles à faible revenu.

\section{Premier thème : enfances et mouvements}

Une soixantaine de portraits d'élèves ont été recueillis au fil de cette étude, dont un peu plus de la moitié à l'école l'Hirondelle. Ces dessins véhiculent tous l'idée d'une présentation de soi à partir d'influences linguistiques et culturelles variées. Nous présentons ici deux récits (portraits de langues) et quelques extraits supplémentaires d'entrevues qui illustrent à la fois des parcours communs dans le cas des élèves de la classe, et singuliers, dans la manière de (se) comprendre, de mettre en lien des situations, de construire des repères au quotidien et de présenter son parcours de vie. Voici d'abord les portraits d'Aya et de Kouma. En réponse à la consigne : « Je dessine sur ma silhouette les langues et les cultures qui m’habitent », voici ce que ces élèves ont partagé.

Aya (élève de la classe de $5^{\mathrm{e}} / 6^{\mathrm{e}}$ année)

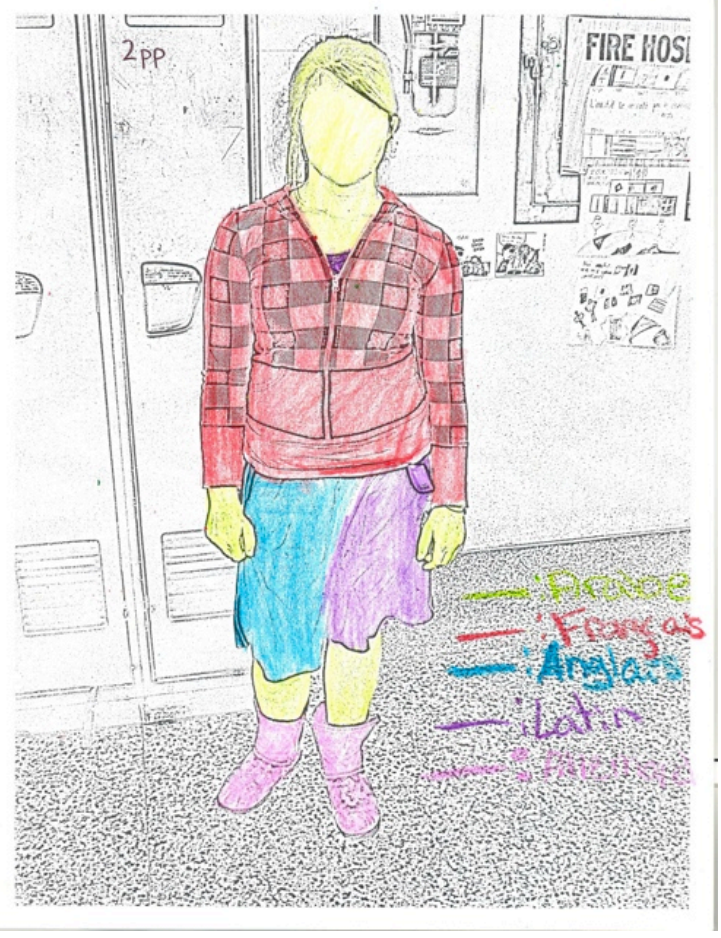

Aya : j'ai colorié ma tête, mes mains et mes pieds en vert, c'est pour l'arabe. Ma chemise en rouge, c'est pour le français. Une partie de mes pantalons en bleu, c'est pour l'anglais, une partie pour le latin est en violet, mes chaussures sont roses pour l'allemand.

\footnotetext{
${ }^{3}$ Des entretiens ont été menés avec la directrice et quatre des cinq membres pionniers du personnel enseignant ainsi qu'avec les deux enseignantes titulaires de la classe de $4 / 5^{\mathrm{e}}$ année et de la classe de $5 / 6^{\mathrm{e}}$ année ayant participées à l'étude.
} 
Chercheure : et pourquoi tu as choisi ces catégories-là ?

Aya : l'arabe, c'est parce que je suis Libanaise, je parle arabe à la maison, je parle français à l'école, et je parle anglais avec mes amis, le latin je sais un petit peu, et l'allemand un peu.

Chercheure : et comment as-tu appris le latin et puis l'allemand?

Aya : le latin, je connais juste une phrase, que madame Dominique (la directrice de l'école) nous a apprise. L'allemand, je savais un peu parce que mes cousins vivent en Allemagne, et mon père est allé en Allemagne une fois, et il m'a appris un peu...

Chercheure : et puis, tu as mis une grande partie de ton dessin en rouge, est-ce qu'il y a une raison pour ça?

Aya : un peu, parce que... [je suis née au] Québec, et on parle français là-bas

Chercheure : est-ce que tu peux me dire, est-ce qu'il y avait des raisons, pourquoi tu as choisi par exemple de mettre le vert ici, à la tête, près des jambes?

Aya : comme ça, c'est ma peau, vert c'est ma couleur préférée, c'est pour le Liban encore parce que le cèdre du Liban sur le drapeau, c'est vert. (entretien avec Issam, Yusra, Elias et Aya, élèves)

À la question de saisir comment les enfants construisent leurs repères quotidiens à travers l'expérience de mouvements migratoires familiaux, on voit paraître ici un lien affectif avec le pays d'origine de la famille (" je suis libanaise "; " vert c'est ma couleur préférée »). Elle ajoute avoir des liens de parenté ailleurs dans le monde en faisant référence à l'Allemagne, avoir des liens avec le Québec et précise que ses interactions au jour le jour se font en contexte plurilingue (" je parle arabe à la maison, je parle français à l'école, et je parle anglais avec mes amis »). Passons au portrait que Kouma nous présente.

\section{Kouma (élève de la classe de $5^{\mathrm{e}} / 6^{\mathrm{e}}$ année)}

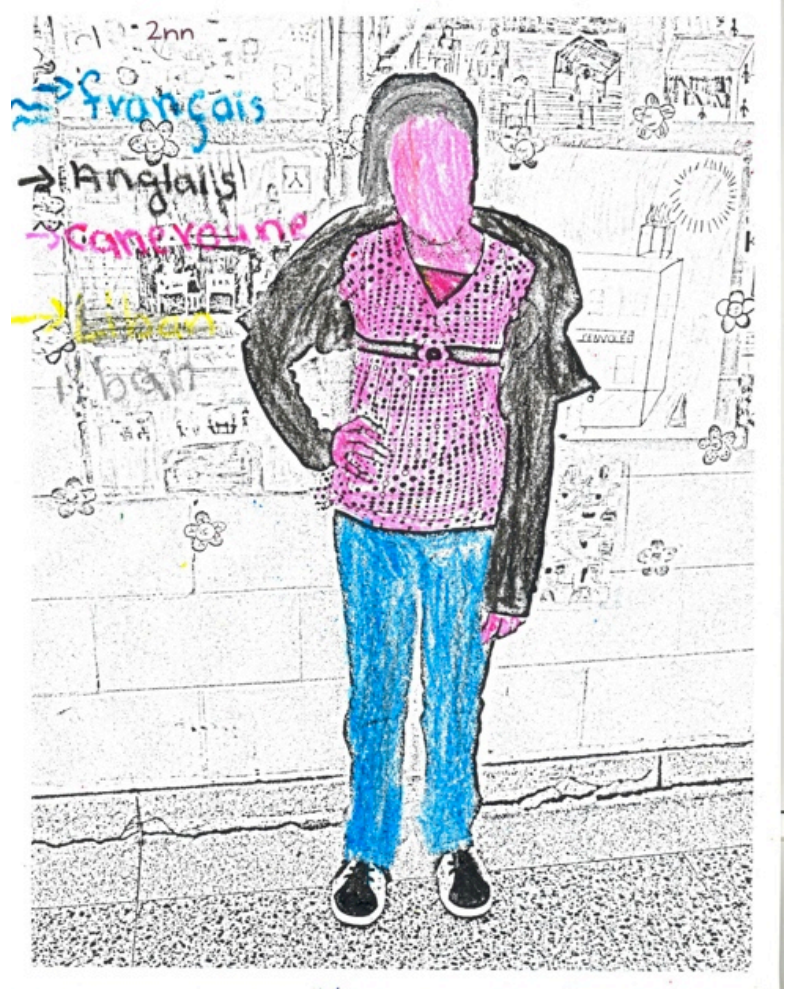


Kouma : j'ai choisi quatre pays, le français en bleu, ça veut dire l'eau et le ciel parce qu'il ne pleut presque pas en Afrique, pas beaucoup. J'ai choisi le noir pour anglais. Le rose pour Cameroun et gris pour Liban. J'ai mis le français, le bleu sur mes pantalons. Le noir sur mon chandail. Puis j'ai mis le rose sur mon chandail et ma face, puis le Liban sur mes cheveux...

Chercheure : ici, tu as mis beaucoup de rose pour le Cameroun ? Ton visage et ton corps aussi ?

Kouma : parce que j'ai fait l'école là-bas et j'ai grandi un peu là-bas. J'ai fait plus de temps avec ma mère que mon père

Chercheure : et ta mère est du Cameroun, c'est ça?

Kouma : oui

Chercheure : parce que tu as mis le Liban aussi?

Kouma : parce que mon père vient du Liban, je n'ai pas eu le temps d'aller voir ma grand-mère et ça c'est pourquoi parce que "I miss her".

Chercheure : donc, c'est pour te rappeler ta grand-mère aussi [...]

Chercheure : finalement, le français tu l'as mis sur tes jambes? Pourquoi tu l'as mis sur tes jambes?

Kouma : parce que... toute ma vie, (j'ai) parlé français et je reviens d’un pays français. (entretien avec Bassem, Marwa, Waël et Kouma, élèves)

Dans cet extrait, Kouma nous présente une vision de la famille qui inclut de manière intégrée ses liens avec le Cameroun et le Liban. Elle fait aussi un lien entre sa scolarisation au Cameroun (« parce que j'ai fait l'école là-bas ») et l'école de langue française en Ontario (sans le mentionner en entrevue, mais du fait de mener cette entrevue alors qu'elle se trouve à l'école française). Elle crée aussi des liens à l'endroit d'une identité francophone en évoquant la continuité ( toute ma vie, [j’ai] parlé français et je reviens d’un pays français »).

Nous avons remarqué que les élèves ont eu recours, assez fréquemment, aux symboles nationaux, soit dans le choix de couleurs (" parce que le cèdre du Liban sur le drapeau, c'est vert ») ou encore dans le dessin (la représentation de drapeaux sur la silhouette) (voir aussi, Farmer, 2012 ; Farmer, Cepin et Breton-Carbonneau, 2015). Ceci peut s'expliquer en raison du fait que l'étude traitait de la mobilité internationale, d'une part, et que le contexte scolaire constitue un des lieux de transmission des idéologies nationales. Par contre, l'agencement des catégories identitaires permet d'illustrer un découpage plus fluide au sein d'espaces et de revoir la classification entre élèves locaux et migrants. Tous les portraits recueillis mettent en valeur un agencement composé d'une grande variété de références linguistiques et culturelles et de valeurs distinctes accordées aux éléments constitutifs. Ceci se voit dans la proportion donnée à une couleur (dans le cadre d'une légende de couleurs) en relation à une autre ou encore dans l'association faite avec les parties du corps (" c'est ma peau, vert, c'est ma couleur préférée »). Ces élèves réfléchissent ainsi à leur parcours de vie en intégrant des éléments importants de leur histoire personnelle et familiale, des éléments qui souvent échappent aux classifications préétablies des chercheurs. De manière plus critique, un tel agencement invite à étudier les conditions sociales et institutionnelles qui donnent lieu à la construction de nouvelles catégories d'inclusion et d'exclusion. Comme tous les récits biographiques, il importe de noter que les dessins constituent un portrait réalisé à un moment particulier et donc un tel portrait est sujet modifications suivant les réflexions, moments et expériences de vie des personnes qui "se racontent". 
Les élèves de l'école l'Hirondelle ont proposé une vision « en contexte » et nuancée des liens culturels et familiaux les reliant à un monde en réseaux. À la continuité des parcours s'allient ainsi la douleur de l'absence et l'éloignement de la famille. Cette idée est ressortie dans de nombreux entretiens, notamment dans le cadre des discussions sur des photos prises par les élèves dans leur quartier et à la maison ${ }^{4}$.

Chercheure : et toi Ashraf, si tu avais à prendre une dernière photo ?

Ashraf : je prendrais une photo de toute ma famille, pas juste ceux qui sont dans notre maison, de toute ma famille, à Montréal, à Toronto et en Australie. (entrevue avec Ashraf et trois élèves de $4^{\mathrm{e}} / 5^{\mathrm{e}}$ année)

Hind : moi aussi, je ferais comme elle [prendre la famille en photo] parce que quand on est tous ensemble, tu sens que tu es très fière, c'est très beau...

Lounis : je prendrai une photo de moi et ma famille, mes grands-pères, mes grands-mères dans la mer, pas dans la mer, une petite rivière, je peux nager, car toute ma famille est au Liban, j'ai personne ici, j'ai juste trois cousins. [...] j'ai juste un grandpère et une grand-mère ici, tout le monde est au Liban... (entrevue avec Yazel, Aïda, Hind et Lounis, élèves de $4^{\mathrm{e}} / 5^{\mathrm{e}}$ année)

Ces échanges nous invitent à réfléchir à l'épaisseur des liens familiaux et sociaux qui se tissent à travers différentes expériences de mobilité et à concevoir le quotidien de l'école comme espace privilégié d'exploration d'enjeux sociaux et politiques qui touchent de près la vie des élèves (Farmer et Prasad, 2014).

\section{Deuxième thème : école et mouvements}

Comment l'école s'adapte-t-elle au renouvellement continu des familles ? Qu'en est-il du découpage entre locaux et migrants? Et quels types de défis l'école doit-elle relever? Afin de trouver des éléments de réponses à ces questions, nous avons organisé la présentation et l'analyse des résultats à partir de trois éléments : la présentation d'éléments biographiques de la directrice et d'une enseignante, l'orientation sociale donnée à l'école et enfin les défis auxquels sont confrontés les acteurs institutionnels de l'école dans la prise en considération de parcours de familles en mouvement.

La francophonie évoquée dans les récits d'élèves s'inscrit ainsi dans la perspective d'une francophonie internationale. Il en va de même des représentations mises de l'avant par la direction d'école et les enseignants. Nous avançons qu'un tel positionnement a joué sur la manière de percevoir les catégories entre élèves locaux et migrants. Commençons par la directrice de l'école, Madame Dominique. Elle se définit comme francophone issue d'un parcours familial international. Sa lecture de la situation est liée à cette conscience de partager une histoire migratoire avec les familles de l'école, à l'idée d'avoir par ailleurs grandi dans le sud-ouest de l'Ontario et enfin d'avoir choisi consciemment de s'entourer d'un personnel provenant des quatre coins du monde et de la région. Elle explique :

Mes grands-parents étaient Hongrois, mes grands-parents étaient des immigrants. Ils sont passés à travers des épreuves. Ils ont très bien réussi dans la vie canadienne. Donc, je voyais mes racines en étant des racines d'immigrantes donc moi, je ressemble à mon personnel (de l'école) même si j'ai grandi à $X$, mes racines sont les racines de nouveaux arrivants parce que ce sont des valeurs que je vis [...] et je ne sais pas, j'ai des membres du personnel de partout. (entretien avec Dominique, directrice)

On voit dans ce discours un premier regard relativiste entre l'ici et l'ailleurs, " je ressemble à mon personnel même si j'ai grandi [ici] ». Ce discours est repris chez les enseignants, ce qu'illustre Madame Lina, enseignante de la classe de $5^{\mathrm{e}} / 6^{\mathrm{e}}$ année.

\footnotetext{
${ }^{4}$ Dans le cadre d'une approche méthodologique intra-site, les élèves ont aussi pris une série de photographies à l'école, dans le quartier et à la maison. Ils ont reçu la consigne suivante : " Je prends en photo des personnes, des objets et des lieux qui me branchent (avec lesquels j'ai développé un lien au courant de ma vie) ». Afin d'ouvrir la discussion en fin d'entrevue, nous avons demandé aux élèves d'indiquer, s'ils avaient une dernière photo à prendre, quelle photo ils prendraient.
} 

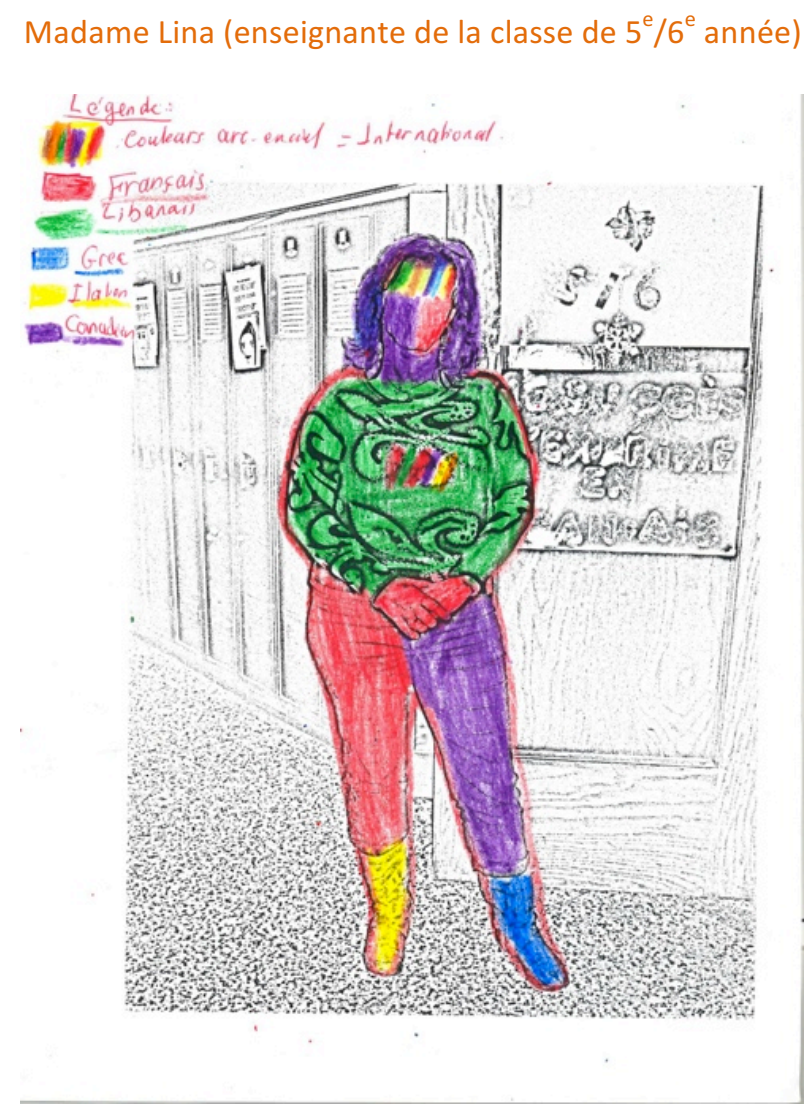

Lina précise qu'elle enseigne depuis 30 ans. Elle a travaillé dans les écoles du Liban principalement et enseigne au Canada depuis 7 ans.

Alors, le front et le cœur sont [la catégorie] internationale. Toutes les couleurs du monde de l'arc-en-ciel. J'ai choisi aussi la couleur rouge qui représente... le français... parce que je suis francophone. J'ai représenté une grande partie de mon corps en rouge et j'ai colorié aussi la moitié de moi-même en vert parce que je suis Libanaise (entretien avec Lina et Emiranda, enseignantes à l'école primaire l'Hirondelle)

Dans cet extrait d'entrevue, il est intéressant de noter que cette enseignante est d'origine libanaise, tout comme un très grand nombre des élèves de l'école. Elle partage ce lien avec eux. Elle fait fréquemment des allers-retours, son conjoint, ses enfants vivent à la fois au Liban et au Canada ${ }^{5}$. Elle évoque une ouverture sur les cultures du monde ("le front et le cœur sont [la catégorie] internationale. ») et une identification à la francophonie. Dans sa représentation de la francophonie et du mouvement, on ne ressent pas une opposition entre l'ici et l'ailleurs, mais plutôt, et de nouveau, un travail de recomposition dans son parcours de vie. Le récit des autres membres du personnel de l'école, qu'ils aient grandi en Ontario ou qu'ils aient un parcours plus international, ressemble beaucoup à celui de Lina.

Nous l'avons évoqué, le paradigme des nouvelles mobilités met de l'avant qu'on ne peut concevoir le mouvement sans tenir compte par la même occasion des lieux d'ancrage. Le leadership mis de l'avant par la direction de l'école primaire l'Hirondelle ainsi que le soutien du personnel enseignant envers une telle vision font de cette école un lieu particulièrement intéressant pour comprendre les enjeux liés à la prise en considération du mouvement des familles par l'école. Plusieurs stratégies mises de l'avant donnent lieu de croire que l'école l'Hirondelle joue un rôle important comme un lieu d'ancrage des familles. Celles-ci incluent notamment des stratégies d'accueil l'ouverture de l'école aux activités communautaires, la diversification des moments de rencontres avec les parents

\footnotetext{
${ }^{5}$ Ce qu'elle partage lors de discussions informelles.
} 
et l'aiguillage des familles vers les services sociaux de la région, un travail fait spontanément par la direction d'école et souvent en réponse à une situation d'urgence.

Et pour soutenir les élèves et le personnel de l'école lors de situations difficiles, la direction de l'école a mis sur pied une classe d'adaptation. Nous aimerions souligner cette initiative en particulier du fait qu'elle aborde les difficultés scolaires non pas à partir d'une vision individualiste et déficitaire de "l'élève en difficulté " ou " en crise » (et sa famille), mais bien au sens de situations liées à un contexte donné et dont personne n'est à l'abri. Nous avançons qu'une telle approche relationnelle peut aider à réduire les effets négatifs d'une vision dichotomique entre élèves locaux et migrants. Elle ne propose pas par ailleurs une vision romantique des familles ou de l'école, mais prévoit plutôt des mécanismes de soutien.

Donc j'ai créé une classe, c'est une classe d'adaptation scolaire... On devrait s'entraider, collaborer et ça devrait être fluide... Chaque personne vit ses moments difficiles dépendant des circonstances... Donc, j'ai toujours envisagé comme une île de la paix si vous voulez, une classe ou on peut juste aller respirer... la chose la plus importante c'est de leur [les élèves] donner un lieu ou ils peuvent juste arriver, se défouler, se calmer et ça, c'est toute sorte de stratégies, est-ce que tu veux une toast... juste t'assoir et bouder... faire un dessin, lire un livre... ? Donc... l'idée c'est que ça soit fluide, que ça soit intégré. Les enseignantes-ressources, [les enseignantes] en salle de classe, peu importe... tout le monde à besoin d'aide ! (entretien avec Dominique, directrice)

L'extrait d'entretien qui suit avec Madame Léa, enseignante spécialisée et Monsieur Philippe, enseignant d'éducation physique et de soutien à l'intégration des élèves, illustre la manière dont la mission sociale de l'école est reprise par le personnel.

Philippe : je trouve qu'ici la plupart des enfants qu'on a ce sont des enfants qui viennent de familles nouvelles au Canada [...] les parents ne sont pas nécessairement stables au niveau de l'emploi même au niveau de l'immigration. Donc, souvent c'est des enfants qui sont ici de passage, qui peuvent aller ailleurs dans d'autres villes, dépendamment vraiment de la stabilité des familles. Si [...] elles ont un emploi ou bien un business qui fonctionne bien, elles restent. Mais si ce sont des familles qui sont nouvelles, qui recherchent encore, souvent elles repartent. On a perdu beaucoup d'enfants qui sont allés à Edmonton $[\ldots]$

Philippe : je pense aussi que l'école subit ça... nous aussi on subit ça.

Léa : le stress et l’anxiété montent.

Chercheure : chez les enfants?

Léa : le comportement change.

Philippe : Oui... le comportement des enfants joue beaucoup aussi parce que s'ils ne sont pas bien à la maison, ils ne vont pas être bien à l'école non plus, ils vont vivre des anxiétés qu'ils amènent de la maison, l'école essaie de les intégrer dans l'école, les accueillir, de leur donner un certain réconfort, le matin, on fait la réception des enfants.

Léa : Le petit déjeuner le matin oui, de leur montrer des stratégies à utiliser. Mais, c'est difficile à expliquer, mais c'est vraiment difficile avec certains élèves, quand leurs amis quittent, ou bien quand il y a de la chicane à la maison, ou quand... il y en a même qui n'ont pas assez d'argent pour la nourriture, il y en a que c'est difficile pour eux. Alors, oui ils viennent à l'école et ça se vide. (entretien avec Léa et Philippe, enseignants)

On voit se dégager la mission sociale de l'école et l'attention aux aléas des parcours migratoires de même que les défis que pose l'établissement dans une ville ouvrière. On se trouve loin ici des mouvements d'élite mondialisés. On connaît encore très peu ce que ce type de migration représente du point de vue des élèves et comment soutenir plutôt que stigmatiser des parcours familiaux marqués par les pressions d'une mobilité précaire. Les acteurs scolaires de l'Hirondelle s'interrogent sur ce sujet, réfléchissent, d'où tout l'intérêt de partager les inquiétudes soulevées en entrevues. Ils ne prônent pas une solution plus qu'une autre, ils cherchent à comprendre ("Mais, c’est difficile à expliquer, mais c'est vraiment difficile avec certains élèves »). 
Malgré les stratégies mises de l'avant au sein de l'école, cette dernière ne peut échapper au fait qu'elle se rattache à un système d'enseignement provincial qui, bien qu'il se soit doté d'une stratégie sur l'équité et l'inclusion (MOE, 2009), ne tienne pas compte du mouvement des familles. Ceci est particulièrement criant dans la manière dont sont évaluées et classées les écoles entre elles. Un des plus grands enjeux pour l'école primaire l'Hirondelle a trait à l'obligation de réaliser des tests prescrits par la province dans les classes de $3^{\mathrm{e}}$ année et de $6^{\mathrm{e}}$ année (tests de I'Office de la qualité et de la responsabilité en éducation, aussi appelé l'OQRE). L'ensemble du personnel y a fait référence spontanément en entrevue. Ces tests désavantagent les élèves de l'école en ne tenant pas compte dans ces examens des connaissances d'élèves exposés à d'autres contenus et manières d'évaluer dans leur parcours mobiles. Par ailleurs, à l'école l'Hirondelle, les familles quittent les classes avant la fin de l'année scolaire en raison des déplacements internationaux au sein des familles durant l'été. L'école se vide, explique-t-on, un peu avant la période des tests de fin d'année. Ceci a aussi un effet d'entraînement sur la réputation de l'école, en ajoutant à une situation déjà précaire l'exode de familles mieux nanties. Sur le plan systémique, la publication du classement des écoles aux tests provinciaux contribue de surcroît à alimenter les préjugés à l'endroit de l'école l'Hirondelle, une école dite de centre-ville accueillant des familles migrantes et dont les résultats au classement viennent renforcer les préjugés dont elle fait l'objet. Mieux comprendre la complexité des nouvelles mobilités dans les parcours familiaux pourrait atténuer le travail de classement qui s'opère actuellement, en soulignant en quoi ces instruments de mesure à grande échelle conduisent aussi à perpétuer les catégories d'inclusion et d'exclusion entre élèves locaux et migrants.

\section{Conclusion}

Ce texte présente les résultats d'une étude menée dans une école de langue française de l'Ontario accueillant des familles à faibles revenus issues de l'immigration. Nous avons cherché à explorer le potentiel que pourrait avoir une analyse reposant sur le paradigme des nouvelles mobilités. Bien que ce cadre ait été développé dans d'autres domaines que celui de l'éducation, nous nous sommes demandé si une analyse qui s'intéresse aux parcours singuliers des acteurs, une analyse qui part du bas -dans cette étude, des élèves eux-mêmes en relation avec les adultes présents dans leur vie - peut offrir de nouvelles perspectives pour comprendre les parcours d'élèves issus de l'immigration et le rôle laissé à l'école dans ces parcours. La conscience qu'ont les élèves, leurs familles et les acteurs de l'école de vivre dans un monde commun peut-elle mener à brouiller les clivages entre locaux et migrants en milieu scolaire ? Et que dire des nouvelles formes de mobilité où se dessinent des mouvements de population plus rapides, fluides et individualisés ? Comment les jeunes se construisent-ils dans ces mouvements ?

L'exploration du paradigme des nouvelles mobilités et l'usage d'approches visuelles réflexives nous ont conduits à mettre en valeur, à travers les portraits d'Aya et de Kouma, le travail d'agencement de catégories identitaires réalisé par les jeunes ainsi que l'idée de continuité dans le mouvement à travers les références à la famille et à l'école. Les analyses ont aussi fait ressortir une vision de la famille étendue non seulement au sens de générations, mais également de réseaux internationaux. Du point de vue des acteurs institutionnels, nous avons aussi observé un travail de recomposition de catégories identitaires dans laquelle la francophonie est comprise à partir des liens internationaux qui rassemblent les acteurs. Nous avons mis de l'avant la mission sociale développée par l'école, en lien avec l'idée d'aborder l'école comme lieu d'ancrage, et nous avons souligné les défis auxquels se trouve confrontée l'école, notamment dans le cadre d'évaluations auxquelles elle est soumise lors des tests provinciaux standardisés.

Cette étude, exploratoire à l'origine, a suscité beaucoup intérêt, se traduisant par une participation deux fois plus élevée que ce qui avait été anticipé. Nous avons cherché à recueillir des paroles d'acteurs, enfants et adultes, aux histoires largement méconnues. Nous avons trouvé d'ailleurs très peu de documentation (tout au moins de l'information publique) au sujet de l'école primaire l'Hirondelle, et rien qui permettrait de relater la profondeur des relations développées entre acteurs au quotidien. Au-delà des résultats de recherche, la dimension réflexive de ce type d'enquête peut aussi aider à remettre en question la catégorisation entre locaux et migrants.

\section{Remerciements}

Nous remercions le Conseil de recherches en sciences humaines du Canada qui a financé cette enquête dans le cadre du programme de Subventions ordinaires (2009-2012). 


\section{Références bibliographiques}

Appadurai, A. (1996). Modernity at Large: Cultural Dimensions of Globalization. Minneapolis : University of Minnesota Press.

Beau, S. et Weber, F. (2003). Guide de l'enquête de terrain. Paris : La Découverte.

Belkhodja, C. (2008). Immigration et diversité au sein des communautés francophones en situation minoritaire. Canadian Issues / Thèmes Canadiens, Printemps.

Bluebond-Langner, M. et Korbin, J. E. (2007). Challenges and opportunities in the anthropology of childhoods: An introduction to "Children, Childhoods, and Childhood Studies". American Anthropologist, 109(2), 241-246.

Bourdin, A. (2005). Les mobilités et le programme de la sociologie. Cahiers internationaux de sociologie, 2005/1 no $118,5-21$.

Busch, B. (2010). School language profiles : Valorizing linguistic resources in heteroglossic situations in South Africa. Language and Education, 24(4), 283-294.

Busch, B., Jardine, A. et Tjoutuku, A. (2006). Language biographies for multilingual learning (vol. 24). Cape Town : PRAESA Occasional Papers.

Castellotti, V. et Moore, D. (2009). Dessins d'enfants et constructions plurilingues. Territoires imagés et parcours imaginés. Dans M. Molinié (éd.), Le dessin réflexif. Éléments pour une herméneutique du sujet purilingue (p. 45-85). Paris : Centre de Recherche Texte Francophonies (CRTF) Encreages, Belles Lettres.

Christensen, P. M., et James, A. (2008). Research with children: perspectives and practices ( ${ }^{\mathrm{e}}$ éd.). Abingdon : Routledge.

Corsaro, W. (2005). The Sociology of Childhood (2 ed.). Thousand Oaks : Pine Forge Press.

Élias, N. (1997). Logiques de l'exclusion (avec J. Scotson). Paris : Fayard.

Faist, T. et Kivisto, P. (dir.). (2007). Dual citizenship in global perspective: from unitary to multiple citizenship. New York : Palgrave Macmillan.

Farmer, D. (2012). Portraits de jeunes migrants dans une école internationale au Canada. La revue internationale de l'éducation familiale, 31, 73-94.

Farmer, D. et Cepin, J. (2015). Researching along with children and youth: Making use of creative visual methods throughout the research process. Dans T. Skelton (directrice de la collection), Geographies of Children and Young People, Major Reference Work, L. Holt et R. Evan (dir. du vol. 2), Methodological Approaches. New Delhi : Springer.

Farmer, D., Cepin, J. et Breton-Carbonneau, G. (2015). Students' Pathways Across Local, National and SupraNational Borders : Representations of a Globalized World in a Francophone Minority School in Ontario, Canada, Journal of Social Sciences Education. 14 (3), 75-83.

Farmer, D. et Prasad, G. (2014). Mise en récit de la mobilité chez les élèves plurilingues : portraits de langues et photos qui engagent les jeunes dans une démarche réflexive. Glottopol, 24, 80-98.

Fass, P. (2007). Children of a New World. New York : New York University Press.

James, A. (2007). Giving Voice to Children's Voices: Practices and Problems, Pitfalls and Potentials. American Anthropologist, 109(2), 261-272.

Heinich, N. (2002). La sociologie de Norbert Elias. Paris : La Découverte.

Kaufmann, V. (2005). Mobilités et réversibilités : vers des sociétés plus fluides ? Cahiers internationaux de sociologie, 118, 119-135.

Kivisto, P. (2001). Theorizing transnational immigration : a critical review of current efforts. Ethnic and Racial Studies, 24(4), 549-577. 
Krumm, H. (2008). Plurilinguisme et subjectivité : Portraits de langues par les enfants plurilingues. Dans G. Zarate, D. Lévy et C. Kramsch (dir.), Précis du plurilinguisme et du pluriculturalisme (p. 109-112). Paris : Éditions des archives contemporaines.

Landri, P. et Newmann, E. (2014). Introduction. Mobile sociologies of education. European Educational Research Journal, 13(1), 493-503.

Ministère de l'éducation de l'Ontario (MEO) (2009). Stratégie ontarienne d'équité et d'éducation inclusive. Ottawa : Gouvernement de l'Ontario.

Mitchell, K. (2003). Educating the national citizen in neoliberal times : From the multicultural self to the strategic cosmopolitan. Transactions of the Institute of British Geographers, 28(4), 387-403.

Montandon, C. (1998). La sociologie de l'enfance: I'essor des travaux en langue anglaise. Éducation et société, 2, 91118.

Ní Laoire, C. Carpena-Méndez, F. Tyrrell, N. et White, A. (2010). Introduction : Childhood and migration - mobilities, homes and belongings. Childhood, 17(2), 155-162.

Nsamenang, A. (2002). Adolescence in Sub-Saharan Africa: An image constructed from Africa's Triple Inheritance. Dans B. Brown, R. Larson et T. Saraswati (dir.), The World's Youth: Adolescence in Eight Regions of the Globe. New York : Cambridge University Press.

Perrenoud, P. (1995). Métier d'élève et sens du travail scolaire ( ${ }^{\mathrm{e}}$ éd.). Paris : ESF éditeur.

Portes, A. (1999). Immigration theory for a new century: some problems and opportunities. Dans C. Hirschman, P. Kasinitz et J. DeWind (dir.), The Handbook of International Migration: The American Experience. New York : Russell Safe Foundation.

Prout, A. (2005). The future of childhood: towards the interdisciplinary study of children. New York : Routledge Falmer.

Ralph, D. et Staeheli, L. (2011). Home and migration : Mobilities, belongings and identities. Geography Compass, 5(7), 517-530.

Rose, G. (2014). On the relation between 'visual research methods' and contemporary visual culture. The Sociological Review, 62(1), 24-46.

Sheller, M., et Urry, J. (2006). The new mobilities paradigm. Environment and Planning, 38, 207-226.

Shin, H. (2012). From FOB to cool : Transnational migrant students in Toronto and the styling of global linguistic capital. Journal of Sociolinguistics, 16(2), 184-200.

Sirota, R. (1993). Le métier d'élève. Revue française de pédagogie, 104, 85-108.

Sirota, R. (1998). L'émergence d'une sociologie de l'enfance: évolution de l'objet, évolution du regard. Éducation et société, 2, 9-34.

Sirota, R. (2006). Éléments pour une sociologie de l'enfance. Rennes : Presses Universitaires de Rennes.

Statisique Canada (2012). Série " Perspectives géographiques », Recensement de 2011 [en ligne]. Ottawa : Statistique Canada. https://www12.statcan.gc.ca/census-recensement/2011/as-sa/fogs-spg/Index-fra.cfm

Song, J. (2010). Language ideology and identity in transnational space : Globalization, migration, and bilingualism among Korean families in the USA. International Journal of Bilingual Education and Bilingualism, 13(1), 2342.

Suarez-Orozco, M. et Qin-Hilliard, D. (2004). Globalization: culture and education in the new millennium. Berkeley : University of California Press.

Suarez-Orozco, M., et Suarez-Orozco, C. (2008). Moving Stories. Du Bois Review: Social Science Research on Race, 4(01), 251-259.

Alterstice - Revue Internationale de la Recherche Interculturelle, vol. 6, $n^{\circ} 1$ 
Thamin, N. (2007). Dynamique des répertoires langagiers et identités plurilingues de sujets en situation de mobilités. Thèse de doctorat, Université Stendhal, Grenoble. http://tel.archives-ouvertes.fr/tel-00288974/fr/

Urry, J. (2000). Sociology beyond societies : mobilities for the twenty-first century. New York: Routledge.

Urry, J. (2005). Les systèmes de la mobilité. Cahiers internationaux de sociologie, 2005-1(118), 23-35.

Vatz Laaroussi, M. (2009). Mobilité, réseaux et résilience. Le cas des familles immigrantes et réfugiées au Québec. Québec : Presses de l'Université du Québec.

Vatz Laaroussi, M. (dir.). (2015). Les rapports intergénérationnels dans la migration. De la transmission au changement social. Québec : Presses de l'Université du Québec.

Weenink, D. (2008). Cosmopolitanism as a form of capital : Parents preparing their children for a globalizing world. Sociology, 42(6), 1089-1106.

White, B. (2014). Quel métier pour l'interculturalisme au Québec ? Dans L. Emongo et B. W. White (dir.), L'interculturel au Québec. Rencontres historiques et enjeux politiques (p. 21-44). Montréal : Presses de I’Université de Montréal. 